\title{
gु \\ Impurity diffusion activation energies in Al from first principles
}

\author{
Darko Simonovic ${ }^{1,2}$ and Marcel H. F. Sluiter ${ }^{2}$ \\ ${ }^{1}$ Materials Innovation Institute, Mekelweg 2, $2628 C D$ Delft, The Netherlands \\ ${ }^{2}$ Department of Materials Science and Engineering, Delft University of Technology, Mekelweg 2, 2628CD Delft, The Netherlands
}

(Received 10 November 2008; revised manuscript received 22 January 2009; published 25 February 2009)

\begin{abstract}
Activation energies for vacancy-mediated impurity diffusion in face-centered-cubic aluminum have been computed $a b$ initio for all technologically important alloying elements, as well as for most of the lanthanides. The so-called five-frequency rate model is used to establish the limiting vacancy interchange process. Many elements were shown to be limited by Al-vacancy interchanges. For these elements we showed that the diffusion activation energy is rather close to that for Al self-diffusion, and additionally the diffusion preexponential factor is of the same order as that for Al self-diffusion. The diffusion activation energy is shown to exhibit a linear relation with the solute partial molar volume in Al. In contrast, transition metals are shown to deviate strongly from these generalities. Diffusion of transition-metal atoms is limited by solute-vacancy interchanges that require remarkably high activation energies. Transition-metal diffusivities in Al show strong trends with the number of $d$-valence electrons but not with partial molar volume.
\end{abstract}

DOI: 10.1103/PhysRevB.79.054304

PACS number(s): 66.30.J-, 61.72.jd, 82.20.Db

\section{INTRODUCTION}

Aluminum alloys are among the most widely applied alloys. The majority of these alloys derive their mechanical properties from precipitation hardening. ${ }^{1}$ For the investigation of kinetic processes in aluminum alloys in the solid state, such as solution treatment and aging, we need a detailed insight in diffusion. Although for diffusion in aluminum much more data are available than for most metals, nevertheless there are so many gaps, in our knowledge, that tendencies and trends are generally poorly known and understood. This is illustrated by the complete absence of any data on the diffusion parameters of rare-earth (RE) elements in $\mathrm{Al}^{2}$

Fortunately, it is now possible to compute many aspects of vacancy-mediated diffusion. ${ }^{3-7}$ Although the foundations on which such $a b$ initio calculations are based are not new, the calculations have become feasible through advances in algorithms $\mathrm{s}^{8,9}$ and computer hardware only recently. The calculations are based on electronic density-functional theory (DFT) (Refs. 10 and 11) coupled with transition state theory (TST) in the harmonic approximation. ${ }^{12,13}$ DFT has already proved to be a remarkably powerful tool to predict and understand the energetics and thermodynamics of alloys. ${ }^{14-17}$ TST has become a practical tool in the context of DFT calculations now that efficient algorithms for finding the minimum-energy path (MEP) have been developed, such as the nudged elastic band (NEB) method. ${ }^{8,9}$ Other ingredients required by TST for computing diffusion parameters are the vibrational frequencies which can be computed $a b$ initio $^{18}$ as well, but at a high computational cost.

In this study, we use $a b$ initio methods and TST to calculate the diffusion activation barrier for a range of alloying elements in the dilute limit, including the rare earths and all currently technologically important alloying elements. The self-diffusion coefficient of aluminum was calculated as well, including the diffusion pre-exponential factor (prefactor). We have also included the effect of vacancy-solute interaction, which causes the vacancy concentration near a sol- ute atom $(X)$ to deviate from the bulk value, through calculation of the vacancy- $X$ binding energy. ${ }^{19}$ Calculations of the vacancy migration transition barriers for vacancy- $X$ and vacancy-Al pairs near a $X$-vacancy pair have been used to examine which of these transitions is rate determining. Knowledge of the rate determining step allows us to estimate the diffusion prefactor within about 1 order of magnitude for those $X$ whose diffusion is limited by vacancy-Al interchanges.

\section{THEORY}

For the computed transport coefficients to be correct the postulated mechanism of transport should be dominant and the transition barrier must be at least several times the thermal energy. In close-packed structures the dominant mechanism in bulk is the vacancy mechanism. The divacancy mechanism can be active at higher temperatures, leading to non-Arrhenius behavior, but this effect in aluminum is usually negligible ${ }^{20}$ compared to single vacancy transition due to high formation energy of divacancies and the low melting temperatures of aluminum. The subtle non-Arrhenius behavior of the self-diffusion activation energy $Q$ observed for aluminum is suggested to be caused by thermal lattice expansion $^{20}$ rather than by the divacancy mechanism. For impurities with strong vacancy binding, the divacancy mechanism cannot be excluded a priori.

In metallurgical applications the vacancy concentration takes equilibrium values at elevated temperatures, with defects as main sources/sinks of vacancies. As a result of this, we can consider the atom-vacancy multicomponent system as a one-component system with a well-defined selfdiffusion coefficient. ${ }^{5,21}$ The connection between macroscopic phenomena of self-diffusion and microscopic transitions of atom vacancy is given by ${ }^{22}$

$$
D^{\mathrm{Al}}=a^{2} f e^{S_{f}^{\square} / k_{B}-H_{f}^{\square} / k_{B} T} \widetilde{\nu} e^{-H_{m}^{\square} / k_{B} T},
$$

where $a$ is the fcc lattice parameter, $f$ is the correlation factor that takes a value of $f=0.78$ (see Ref. 23) for the fcc struc- 
ture, $S_{f}^{\square}$ and $H_{f}^{\square}$ are vacancy formation entropy and enthalpy, $H_{m}^{\square}$ is the vacancy migration enthalpy, i.e., the energy barrier to be overcome for an atom to jump into the vacancy, and $\widetilde{\nu}$ is the frequency prefactor of the transition rate. The vacancy formation entropy is given by ${ }^{12}$

$$
e^{S_{f}^{\square} / k_{B}}=\frac{\prod \nu_{i}^{\mathrm{Al}_{N} \square}}{\prod \nu_{i}^{\mathrm{Al}_{N}}},
$$

where the product runs over all relevant vibrational modes of the two systems at minimum energy, with vacancy $\left(\mathrm{Al}_{N} \square\right)$ and without $\left(\mathrm{Al}_{N}\right)$, and $N$ is the number of atoms in the crystal.

Within the same approximations the frequency prefactor of the transition rate is given by ${ }^{13}$

$$
\tilde{\nu}=\frac{\prod \nu_{i}^{\mathrm{Al}_{N} \square}}{\prod^{\prime} \nu_{i}^{\mathrm{Al}_{N} \square \uparrow} .}
$$

This enthalpy is computed as the difference between the energy of the stable initial minimum-energy configuration $\left(\mathrm{Al}_{N} \square\right)$ and the energy of the activated transition state, the saddle-point energy configuration $\left(\mathrm{Al}_{N} \square \uparrow\right)$. Vibrational frequencies $\nu$ are given for those same two configurations: stable initial minimum-energy configuration $\left(\mathrm{Al}_{N} \square\right)$ and the energy of the activated transition state, i.e., the saddle-point energy configuration $\left(\mathrm{Al}_{N} \square \uparrow\right)$. These configurations are used also to evaluate $H_{f}^{\square}$ and $H_{m}^{\square}$. The difference between Eqs. (2) and (3) is that in the denominator of Eq. (3) the imaginary frequency associated with the upward energy curvature of the saddle-point configuration is specifically excluded from the product. This is symbolically indicated by marking the product with a prime. A consequence of the exclusion of the imaginary frequency is that the prefactor $\tilde{\nu}$ has the dimension of frequency, unlike $e^{S_{f}^{\square} / k_{B}}$ which is dimensionless. In principle the frequencies can be obtained from the eigenvalues of the force-constant matrix of a large enough segment of a crystal in real space. The much more efficient dynamical matrix method coupled with small supercells $^{18}$ can be used by assuming periodicity of the (single site) defects and by imposing a finite spatial range of the force constants. This is the method we will apply here.

The transport coefficient in a multicomponent system consisting of two atomic species plus vacancies as mediators of jumps can be represented as a $3 \times 3$ matrix. In the limit of dilute solution and at equilibrium vacancy concentration, there will be one relevant diffusion coefficient for the solute $X$ only. ${ }^{21}$ Our calculations pertain to this limit. If $X$ features metallic bonding and does not break the close-packed character of the Al matrix, the diffusion will be mediated through vacancy jumps. We will limit our study to such substitutional solutes.

If $\square$-X interchanges were the only kind of interchanges that occur, solute atoms would move back and forth and no diffusion would take place. Therefore, other elementary interchanges need to be considered also, such as those involving the $\mathrm{Al}$ atoms that are next to the vacancy of the $\square$ - $X$ pair. These aluminum-vacancy (Al- $\square$ ) transition rates generally

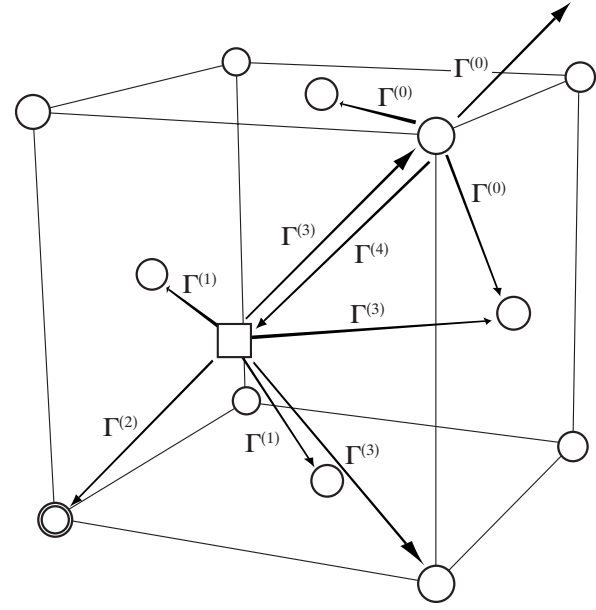

FIG. 1. Transition rates $\Gamma$ for vacancy-atom interchanges in fcc crystals. Circles (double circle) represent $\mathrm{Al}(X)$, square indicates vacancy. Redrawn from Ref. 24.

differ from Al- $\square$ transition rates further away from the solute atom, but they follow the Arrhenius dependence with migration enthalpy for each transition $H_{m}^{\square}$. The same holds true for the formation of vacancy near solute. The energy required to form a vacancy next to an $X$ atom $H_{f}^{\square @ X}$ generally differs from the formation energy of a vacancy in bulk $H_{\square}^{f}$. Previous work ${ }^{19}$ has revealed that these energies differ significantly when the solute and the vacancy are first nearest neighbors only. A convenient quantity in this context is the solute-vacancy binding energy defined as

$$
H_{\text {bind }}^{\square @ X}=H_{f}^{\square}-H_{f}^{\square @ X} .
$$

When an Al- $\square$ interchange moves the vacancy away from the solute, the solute-vacancy binding energy is lost, thus resulting in unequal transition barriers in forward and backward directions. The interplay of the interchanges, including the vacancy jumps in bulk, determines the macroscopic solute diffusion. This can be evaluated within the five-frequency rate model. ${ }^{24}$ Parameters needed for this model are enthalpy of vacancy near solute atom and transition rates of $X$-vacancy $\Gamma^{(2)}$ interchange, Al-vacancy interchange with the vacancy remaining a nearest neighbor of $X \Gamma^{(1)}$, Al-vacancy interchange with the vacancy moving away from $X \Gamma^{(3)}$, in the opposite direction an Al-vacancy interchange with the vacancy moving toward $X \Gamma^{(4)}$, and an Al-vacancy interchange in the bulk $\Gamma^{(0)}$. These five interchanges are depicted schematically in Fig. 1. The five-frequency rate model may give a level of accuracy that is unnecessary, given that transition rates near an $X$ atom may differ by orders of magnitude because as a thermally activated process, it follows an exponential temperature and activation energy dependence. Furthermore, the Arrhenius type of dependence, readily available in experiments, will be hidden in the model by the rather complicated formulas. Keeping the dominant exponential term in the five-frequency rate model gives a better understanding. When the $X-\square$ transition is not the fastest interchange, there will be enough Al- $\square$ interchanges be- 
tween two subsequent $X-\square$ interchanges to wipe out their correlation so that

$$
D=a^{2} \widetilde{\nu} e^{S_{f}^{\square} / k_{B}} e^{-\left(H_{m}^{X}+H_{f}^{\square @ X}\right) / k_{B} T} .
$$

On the other hand if $X-\square$ interchanges occur much more frequently than other Al- $\square$ interchanges near the solute, it will not contribute to the macroscopic transport of solute $X$. The fastest of the two Al- $\square$ interchanges $\left(\Gamma^{(1)}\right.$ and $\left.\Gamma^{(3)}\right)$ would dominate, leading to,

$$
D=f^{5 \omega} a^{2} \widetilde{\nu} e^{S_{f}^{\square} / k_{B}} e^{-\left[\min \left(H_{m}^{\mathrm{Al}(1)}, H_{m}^{\mathrm{Al}(3)}\right)+H_{f}^{\square @ X}\right] / k_{B} T},
$$

where $H_{m}^{\mathrm{Al}(1)}$ is the energy for vacancy migration around the solute $\left(\Gamma^{(1)}\right.$ in Fig. 1) and $H_{m}^{\mathrm{Al}(3)}$ is the energy for vacancy migration away from the solute $\Gamma^{(3)}$. The correlation factor $f^{5 \omega}$ then takes a value between $f^{5 \omega}=1$ and $f^{5 \omega}=3.5$ (see Ref. 24) and it is always larger than the correlation factor for self-diffusion. The vibrational frequencies $\widetilde{\nu}$ are selected in accord with the lowest vacancy migration energy. Summarizing, conventionally expressing $D=D_{o} e^{Q / k_{B} T}$, we will use an activation energy for solute diffusion given by

$$
Q=\max \left[H_{m}^{X}, \min \left(H_{m}^{\mathrm{Al}(1)}, H_{m}^{\mathrm{Al}(3)}\right)\right]+H_{f}^{\square @ X}
$$

and a diffusion prefactor given as

$$
D_{o}= \begin{cases}a^{2} \widetilde{\nu} e^{S_{f}^{\square} / k_{B}} & H_{m}^{X}>\min \left(H_{m}^{\mathrm{Al}(1)}, H_{m}^{\mathrm{Al}(3)}\right) \\ f^{5 \omega} a^{2} \widetilde{\nu} e^{S_{f} / k_{B}} & H_{m}^{X}<\min \left(H_{m}^{\mathrm{Al}(1)}, H_{m}^{\mathrm{Al}(3)}\right) .\end{cases}
$$

\section{METHOD}

In our $a b$ initio calculations periodic supercells are used with one solute atom $X$ and/or one vacancy per supercell. With such a supercell, the solute and vacancy concentrations are much larger than in actual materials. Hence, spurious $X$ - $X$ and $\square$ - $\square$ interactions pollute our computed energies. In metals, where localized charges are effectively screened, interactions between neighboring supercells, i.e., the defects $X$ contained within them, are generally of an elastic character. As the elastic energy density decays as the sixth power of the distance, even quite small supercells can give meaningful results. ${ }^{25}$ While it is possible to correct for spurious interaction effects by extrapolating to infinite supercell size, ${ }^{26}$ here we make corrections through elasticity theory. ${ }^{25}$ In supercell calculations one can typically either impose constant volume for computing energy differences or impose constant, i.e., zero, pressure for computing enthalpy differences. The present case involving a single $X$ in a large piece of material does not match well with either condition when we view it as a single supercell embedded in an aluminum crystal without any defects. Working at constant volume, we assume that all the elastic energy associated with the solute is restricted to the supercell only, with none of that elastic energy being in the embedding perfect crystal. On the other hand, if we impose zero pressure on the supercell, we assume that the embedding medium is infinitely compliant and absorbs all elastic strain. Working with a supercell where the strain energy is divided between the supercell and the surrounding embedding crystal is not straightforward. Within the context of iso- tropic continuum linear elasticity theory it is possible to compute how the elastic energy is shared between the supercell and the surrounding perfect crystal. Al is well represented as an elastically isotropic medium because of the near-unity anisotropy ratio $A=1.14$. Therefore, we followed the description in Ref. 25 to correct for the finite size of the supercell. Starting from Eq. 18 in Ref. 25 and expanding the elastic energy up to and including the second order in the volume, we obtain for the elastic correction to the supercell energy

$$
\Delta E_{\text {elast }}=-\frac{3 V_{0} P_{0}^{2}}{3 B+16 \mu},
$$

where $V_{0}$ is the equilibrium volume of the pure $\mathrm{Al}_{64}$ supercell, $P_{0}$ is the pressure that occurs in the structurally optimized supercell at volume $V_{0}$ where a single $\mathrm{Al}$ atom has been replaced by an impurity, $B$ is the bulk modulus, and $\mu$ is the usual Lamé coefficient. As in Ref. 25 we adopt $B$ $=82 \mathrm{GPa}$ and $\mu=31 \mathrm{GPa}$. In all cases considered the elastic corrections were less than $4 \mathrm{~kJ} / \mathrm{mol}$ impurity atom. The isotropic assumption made here can be overcome through the elegant formulation in reciprocal space by Daw. ${ }^{27}$

The enthalpy of formation of a vacancy in bulk aluminum can be evaluated with

$$
H_{f}^{\square}=H\left(\mathrm{Al}_{N-1} \square\right)-\frac{N-1}{N} H\left(\mathrm{Al}_{N}\right) .
$$

In order to minimize the effect of systematic errors in the $a b$ initio calculations, we assure that whenever energy differences are computed, supercell geometries, $k$-point grids, and other computational settings are the same. In a completely similar manner we define the enthalpy associated with the formation of a vacancy near a solute atom $X$,

$$
H_{f}^{\square @ X}=H\left(\mathrm{Al}_{N-2} X \square\right)-H\left(\mathrm{Al}_{N-1} X\right)+\frac{1}{N} H\left(\mathrm{Al}_{N}\right) .
$$

Electronic DFT calculations within the generalized gradient approximation of Perdew-Burke-Ernzerhof (PBE) type ${ }^{28}$ were carried out with the Vienna $a b$ initio simulation package (VASP). ${ }^{29,30}$ We selected pseudopotentials of the projector augmented wave (PAW) type. ${ }^{31,32}$ The recommended standard potentials ${ }^{33}$ were used; in a few cases we opted for harder potentials with semicore states treated as valence states, Bi_d, Ga_d, Ge_d, Hf_pv, Li_sv, Mo_pv, Nb_pv, Pb_d, Sc_sv, Sn_d, Sr_sv, Ta_pv, Ti_sv, W_pv, Y_sv, and $\mathrm{Zr} \_$sv. For the lanthanides we selected potentials with three $f$-like electrons treated as core states, Ce, Er, Gd, Ho, Lu, $\mathrm{Nd}, \mathrm{Pm}, \mathrm{Pr}, \mathrm{Sm}, \mathrm{Tb}$, and Tm, or two $f$-like electrons treated as core states, Eu. Recently, Gao et al. ${ }^{34}$ carefully examined the PAW-PBE potentials for the rare earths with regard to structural ground-state properties and aluminide compound formation, and the currently selected potentials were found to generally give results that agreed with experiment. Supercells have fcc geometry with $4 \times 4 \times 4$ lattice points with 64 atoms per unit cell in the absence of vacancies (see Fig. 1 of Ref. 25), unless specified otherwise. The primitive translations for such a cell are $a_{\mathrm{fcc}} \times\left[\begin{array}{lll}2 & 2 & 0\end{array}\right]$. This supercell size is a compromise between accuracy and speed, see Table I. Er- 
TABLE I. Vacancy formation enthalpy $\left(H_{f}^{\square}\right)$, computed with Eq. (10), and vacancy migration enthalpy $\left(H_{m}^{\square}\right)$ as function of supercell size as indicated by primitive translations. All calculations used 7 $\times 7 \times 7 k$-point grids.

\begin{tabular}{|c|c|c|}
\hline Supercell size & $\begin{array}{c}H_{f}^{\square} \\
(\mathrm{kJ} / \mathrm{mol})\end{array}$ & $\begin{array}{c}H_{m}^{\square} \\
(\mathrm{kJ} / \mathrm{mol})\end{array}$ \\
\hline$a_{\mathrm{fcc}} \times\left[\begin{array}{lll}1 \frac{1}{2} & 1 \frac{1}{2} & 0\end{array}\right]$ & 77.9 & 90.4 \\
\hline$a_{\mathrm{fcc}} \times\left[\begin{array}{lll}2 & 2 & 0\end{array}\right]$ & 71.9 & 49.3 \\
\hline$a_{\mathrm{fcc}} \times\left[2 \frac{1}{2} 2 \frac{1}{2} 0\right]$ & 68.6 & 51.5 \\
\hline
\end{tabular}

rors associated with the finite size of the supercell and the coarseness of $k$-point sampling are expected to be up to 3 $\mathrm{kJ} / \mathrm{mol}$ for the vacancy enthalpy and transition barrier considered separately, but only $1 \mathrm{~kJ} / \mathrm{mol}$ for diffusion activation barrier. In VASP the setting "Precision=high" was employed. In all calculations, the electronic wave functions were expanded in terms of plane waves up to a cutoff kinetic energy of at least $300 \mathrm{eV}$. Real-space projectors were not used for final static calculations. The convergence criteria for energy and force were $0.1 \mathrm{meV}$ and $10 \mathrm{meV} / \mathrm{nm}$, respectively. Structural optimizations were reinitiated at least twice. Integrations in reciprocal space used the special $k$-point technique of Monkhorst and Pack. ${ }^{35}$ Results relevant to vacancies for various $k$-point grids are listed in Table II. For structural relaxation of atomic positions a grid of $5 \times 5 \times 5 k$ points was found adequate, but an additional static calculation was performed with $7 \times 7 \times 7 k$ points to obtain a more accurate energy. As Table II shows, these $k$-point grids appear to give energies that are converged within $1 \mathrm{~kJ} / \mathrm{mol}$.

The nudged elastic band method with climbing image ${ }^{8,9}$ is used to find the saddle-point configuration. While finding the saddle point the MEP is calculated also.

All data presented are for the $\mathrm{Al}$ fcc lattice parameter held at $0.4085 \mathrm{~nm}$, unless noted otherwise. This value of the lattice parameter corresponds to a temperature of about $630 \mathrm{~K}$. In order to evaluate the prefactor $D_{0}$, vibrational frequencies $\nu$ from Eqs. (2) and (3) need to be found. The dynamical matrix is evaluated $a b$ initio with the direct method ${ }^{36-38}$ using ionic displacements of $0.005 \mathrm{~nm}$.

\section{RESULTS AND DISCUSSION}

The vacancy formation enthalpy depends on the lattice parameter which in turn varies with the temperature. Table

TABLE II. Computed vacancy formation enthalpy $\left(H_{f}^{\square}\right)$ Eq. (10) and vacancy migration enthalpy $\left(H_{m}^{\square}\right)$ for a $4 \times 4 \times 4$ fcc supercell (64 atoms) as a function of the $k$-point grid.

\begin{tabular}{lcc}
\hline \hline$k$-point grid & $\begin{array}{c}H_{f}^{\square} \\
(\mathrm{kJ} / \mathrm{mol})\end{array}$ & $\begin{array}{c}H_{m}^{\square} \\
(\mathrm{kJ} / \mathrm{mol})\end{array}$ \\
\hline $3 \times 3 \times 3$ & 88.6 & 48.9 \\
$5 \times 5 \times 5$ & 64.3 & 50.9 \\
$7 \times 7 \times 7$ & 71.9 & 49.3 \\
$9 \times 9 \times 9$ & 71.6 & 50.4 \\
\hline \hline
\end{tabular}

TABLE III. Computed vacancy formation enthalpy $\left(H_{f}^{\square}\right)$ and computed vacancy migration enthalpy $\left(H_{m}^{\square}\right)$ as a function of the lattice parameter $a_{\mathrm{fcc}}$ and the temperature at which this lattice parameter is attained in actual $\mathrm{Al}$ (Ref. 60) in experiments.

\begin{tabular}{lccc}
\hline \hline $\begin{array}{l}a_{\mathrm{fcc}} \\
(\mathrm{nm})\end{array}$ & $\begin{array}{c}T_{\text {eff }} \\
(\mathrm{K})\end{array}$ & $\begin{array}{c}H_{f}^{\square} \\
(\mathrm{kJ} / \mathrm{mol})\end{array}$ & $\begin{array}{c}H_{m}^{\square} \\
(\mathrm{kJ} / \mathrm{mol})\end{array}$ \\
\hline 0.4040 & 0 & 61.7 & 57.3 \\
0.4050 & 300 & 65.6 & 53.3 \\
0.4085 & 630 & 71.9 & 49.3 \\
0.4120 & 890 & 77.2 & 45.5 \\
\hline \hline
\end{tabular}

III shows the computed vacancy formation enthalpy and the vacancy migration enthalpy as a function of the lattice parameter. In the experimentally relevant temperature range of $630 \mathrm{~K}$, the vacancy formation enthalpy in pure $\mathrm{Al}$ is calculated to be $71.9 \mathrm{~kJ} / \mathrm{mol}$. This agrees well with the experimental values which range from 58 to $74 \mathrm{~kJ} / \mathrm{mol} .{ }^{39}$ An increase in the lattice parameter increases the vacancy formation enthalpy; however it reduces the vacancy migration enthalpy. These changes largely cancel each other, so that the effect of thermal expansion is not quite as strong as one might expect on the basis of either formation enthalpy or migration enthalpy alone. Table III suggests that a lattice expansion corresponding to a $300 \mathrm{~K}$ temperature increase changes the activation energy for diffusion by $2 \mathrm{~kJ} / \mathrm{mol}$. At a lattice parameter of $0.4085 \mathrm{~nm}$ we find the self-diffusion activation energy of $\mathrm{Al}$ to be $71.9+49.3=121.2 \mathrm{~kJ} / \mathrm{mol}$. This value compares well with the experimentally reported values which range between 120.4 and $144.4 \mathrm{~kJ} / \mathrm{mol}^{40-45}$

It is known that both GGA and LDA functionals tend to underestimate the surface energy. ${ }^{46}$ This fact was recently used to correct the vacancy formation energy ${ }^{47}$ and vacancy migration energy. ${ }^{4}$ The main drawback of this correction arises from the difficulty to properly define the surface associated with a vacancy. In the present study we did not use any surface correction term because we found that both the vacancy formation energy and the self-diffusion activation energy of $\mathrm{Al}$ are in fair agreement with experimental data.

The frequency prefactor $\widetilde{\nu}$ in Eq. (3) depends on the change in vibrational modes only, so it is tempting to try to approximate that change using vibrations of only a limited number of ions around the transition given that these calculations are computationally very demanding. As we do not consider periodicity here, these calculations correspond only to the $\Gamma$ point in the dispersion. Table IV shows $\widetilde{\nu}$ when $N$ nearest atoms to the transition path are considered in the 63 atom supercell. It is evident that $\widetilde{\nu}$ does not converge rapidly with the number of atoms $N$. Extrapolation can be done by plotting $\widetilde{\nu}$ versus $1 / N$ and extrapolating to zero, as suggested for entropic terms also. ${ }^{26}$ This gives a value for $N=\infty$ of 16 THz. Data used for such an extrapolation are not quite consistent because for the larger $N$ values, atoms at the boundary are shared between the periodic supercells and their motion is thus restricted. Using Eq. (1) and the value of entropy $S_{f}^{\square}=1.2 k_{B}$ calculated elsewhere, ${ }^{3,4}$ we get a prefactor for selfdiffusion of $D_{0}=0.07 \times 10^{-4} \mathrm{~m}^{2} \mathrm{~s}^{-1}$, in good agreement with other theoretical work $\left(D_{0}=0.24 \times 10^{-4} \mathrm{~m}^{2} \mathrm{~s}^{-1}\right.$ extrapolated 
TABLE IV. Frequency prefactor $\widetilde{\nu}$ associated with vacancy migration as a function of the number of atoms $N$ within a distance $R$ around the Al- $\square$ interchange transition path. $N=63$ corresponds to the whole supercell.

\begin{tabular}{lcc}
\hline \hline$N$ & $\begin{array}{c}R \\
(\mathrm{~nm})\end{array}$ & $\begin{array}{c}\tilde{\nu} \\
(\mathrm{THz})\end{array}$ \\
\hline 1 & 0.14 & 2.23 \\
5 & 0.24 & 3.68 \\
7 & 0.31 & 3.71 \\
9 & 0.33 & 5.04 \\
13 & 0.36 & 5.27 \\
17 & 0.39 & 8.72 \\
29 & 0.50 & 12.08 \\
63 & 0.64 & 14.12 \\
\hline \hline
\end{tabular}

from Fig. 3 of Ref. 3 and $D_{0}=0.066-0.078 \times 10^{-4} \mathrm{~m}^{2} \mathrm{~s}^{-1}$ in Ref. 4) and in fair agreement with experimental results, ${ }^{40-45,48}$ which range from 0.035 to $1.7 \times 10^{-4} \mathrm{~m}^{2} \mathrm{~s}^{-1}$, see Table IV.

The main results of the present study are given in Table $\mathrm{V}$. The elements have been ordered according to the Mendeleev number of Pettifor ${ }^{49,50}$ to highlight trends. The solutevacancy binding energy, the vacancy migration energy, and the diffusion activation energy are seen to all vary strongly with solute species. The binding enthalpies compare reasonably well with those calculated by Wolverton, ${ }^{19}$ where available (Fig. 2). The most noticeable discrepancies are observed for $\mathrm{Ti}, \mathrm{Zr}$, and $\mathrm{Cr}$. It seems that for $\mathrm{Ti}$ and $\mathrm{Zr}$, a large number of $k$ points is essential. We verified our value of the solutevacancy binding enthalpy by using a denser $9 \times 9 \times 9 k$-point grid and by using a larger supercell with 125 atoms while retaining the $7 \times 7 \times 7 k$-point grid. These calculations gave essentially no change in the vacancy binding enthalpy. On the other hand, when we lowered the number of $k$ points to $5 \times 5 \times 5$ in the 64 atom cell we reproduced the results of Wolverton ${ }^{19}$ for $\mathrm{Ti}$ and $\mathrm{Zr}$. The discrepancy observed for $\mathrm{Cr}$ arises from the apparent neglect of spin polarization in the aforementioned study. ${ }^{19}$ The effect of spin polarization in $\mathrm{Cr}$, $\mathrm{Mn}$, and $\mathrm{V}$ is summarized in Table VI. The difference between the spin-polarization enthalpy changes for the $\mathrm{Al}_{63} \mathrm{Cr}$ and $\mathrm{Al}_{62} \square \mathrm{Cr}$ configurations indicates that spin polarization makes $H_{\text {bind }}^{\square @ X}$ about $15.9-11.3=4.6 \mathrm{~kJ} / \mathrm{mol}$ less repulsive. Spin polarization also decreases the energy barrier for $\mathrm{Cr}$ - $\square$ interchange; the initial state is lowered by $15.9 \mathrm{~kJ} / \mathrm{mol}$ while the transition state is lowered by $52.8 \mathrm{~kJ} / \mathrm{mol}$, decreasing the barrier by $36.9 \mathrm{~kJ} / \mathrm{mol}$. A similar reduction in the $\mathrm{Cr}-\square$ interchange barrier was found by Sandberg and Holmestad ${ }^{6}$ and by Mantina $^{7}$ using local spin-density approximation $($ LSDA $)+U$ formalism. $\mathrm{Mn}$ is affected by spin polarization analogously. For Mn spin polarization makes the vacancy binding enthalpy $19.7-16.3=3.4 \mathrm{~kJ} / \mathrm{mol}$ less repulsive. This relatively small difference explains that the earlier neglect ${ }^{19}$ of spin polarization did not cause as large a discrepancy with the current results for Mn. The barrier for $\mathrm{Mn}-\square$ interchange, just like the $\mathrm{Cr}-\square$ interchange, is strongly affected by spin polarization; however, the barrier is lowered by $76.3-19.7=56.6 \mathrm{~kJ} / \mathrm{mol}$ because spin polarization lowers the energy of the transition state more than the energy of the initial state as is apparent also from Table IV when comparing the rows for $\mathrm{Mn}$ and $\mathrm{Mn}^{(M)}$. Sandberg and Holmestad $^{6}$ and Mantina ${ }^{7}$ found a comparable reduction in the barrier for Mn- $\square$ interchange due to spin polarization. Hoshino et $a l .{ }^{51}$ reported that spin polarization lowered the Cr- $\square$ and Mn- $\square$ repulsions, but their neglect of relaxation resulted in a much larger effect. According to our calculations the vanadium solute is spin polarized in the vicinity of a vacancy only. The vacancy binding enthalpy change due to spin polarization of $\mathrm{V}$ is small. However, the energy barrier of $\mathrm{V}-\square$ interchange is reduced by $15.0-0.5=14.5 \mathrm{~kJ} / \mathrm{mol}$.

Figure 2 indicates three regions where strong vacancy binding occurs: (1) large earth alkaline atoms such as $\mathrm{Ca}$ and Sr, (2) early lanthanides such as La and Ce, and (3) heavy semimetals such as $\mathrm{Bi}$ and $\mathrm{Pb}$. Solutes that have strong vacancy binding might strongly affect the precipitation behavior of other alloying elements because of vacancy trapping after the solutionizing heat treatment. The very strong vacancy binding of Sr, causing a vanishing of the vacancy formation energy next to a Sr solute, is remarkable, and at first consideration it appears strange. However, the solution energy for $\mathrm{Sr}$ is very large also, about $102 \mathrm{~kJ} / \mathrm{mol},{ }^{25}$ because this large atom does not fit in the Al lattice. Dissolved in the Al matrix, a Sr solute atom takes a volume that is about 1.75 times larger than that of an $\mathrm{Al}$ atom. ${ }^{25}$ Therefore, the $\mathrm{Sr}$ atom forms a complex with a vacancy in which the $\mathrm{Sr}$ atom is displaced toward the vacancy. The barrier for trading places with the vacancy $H_{m}^{X-\square}$ is very low also because the $\mathrm{Sr}$ atom sits already almost at the midpoint. However, as we mentioned in Sec. I, Sr is not necessarily a very fast diffuser, as the Sr- $\square$ oscillations do not themselves contribute to diffusion. Sr diffusion is actually limited by Al- $\square$ interchanges that, due to the strong distortions around the vacancy with $\mathrm{Sr}$, differ considerably from Al- $\square$ interchanges in pure $\mathrm{Al}$, as is readily seen from $H_{m}^{\mathrm{Al}(1)}$ and $H_{m}^{\mathrm{Al}(3)}$ in Table $\mathrm{V}$.

Other large atoms, such as $\mathrm{Ca}, \mathrm{La}, \mathrm{Ce}, \mathrm{Pb}$, and $\mathrm{Bi}$, behave similar to Sr; they exhibit strong vacancy binding and their diffusion rate is determined by Al- $\square$ interchanges in the immediate vicinity of the solute also. Accordingly, Fig. 3 shows a positive correlation between vacancy binding and partial molar volume of the solute relative to that of the partial molar volume of $\mathrm{Al}$ as is evident for the series $\mathrm{Cu}-\mathrm{Ge}, \mathrm{Pd}-\mathrm{Sb}$, Pt-Bi, and La-Lu.

In line with this is the observation that elements from the sixth row (connected by dashed lines) tend to have larger $H_{\text {bind }}^{X-\square}$ values than elements from the same column from the fifth and fourth rows, with the value from the fifth row being larger than that from the fourth row, because elements at the bottom of a column tend to have larger volumes than elements at the top, as can be seen by following the dasheddotted lines in Fig. 2. The lanthanides completely follow these trends of $H_{\text {bind }}^{X-\square}$ versus atomic volume, with the exception of Eu. With a precisely half-filled f-band, the chemistry of divalent $\mathrm{Eu}$ is also known to differ substantially from the other lanthanides which prefer the trivalent state. It can be recognized in the ordering of the elements according to the Mendeleev number of Pettifor, ${ }^{49,50}$ where $\mathrm{Eu}$ is placed between $\mathrm{Yb}$ and $\mathrm{Sc}$. 
TABLE V. Solute-vacancy binding energy $H_{\text {bind }}^{\square @ X}$, solute migration energies $H_{m}^{X}$, Al migration energies $H_{m}^{\mathrm{Al}(1)}$ for moving an $\mathrm{Al}$ atom around the solute, $H_{m}^{\mathrm{A}(3)}$ for moving an $\mathrm{Al}$ atom toward the solute, (see $\Gamma^{(1)}$ and $\Gamma^{(3)}$ in Fig. 1), diffusion activation energy $Q$ as computed with Eq. (7) and as experimentally measured, all in units of $\mathrm{kJ} / \mathrm{mol}$, and experimental values of the diffusion prefactor $D_{0}$ in units of $10^{-4} \mathrm{~m}^{2} \mathrm{~s}^{-1}$. The computed results pertain to an $\mathrm{Al}$ bulk lattice parameter of $0.4085 \mathrm{~nm}$ that occurs at a temperature of $630 \mathrm{~K}$ as discussed in text. Solutes whose diffusion in $\mathrm{Al}$ is dominated by Al-vacancy interchanges are marked with an asterisk $(*)$. Spin-polarized results for $\mathrm{Cr}, \mathrm{Mn}$, and $\mathrm{V}$ are labeled with a superscript $(M)$.

\begin{tabular}{|c|c|c|c|c|c|c|c|c|}
\hline \multirow[b]{2}{*}{ Solute } & \multicolumn{5}{|c|}{ Calculated } & \multicolumn{3}{|c|}{ Experimental } \\
\hline & $H_{\text {bind }}^{\square @ X}$ & $H_{m}^{X}$ & $H_{m}^{\mathrm{Al}(1)}$ & $H_{m}^{\mathrm{Al}(3)}$ & $Q$ & $Q$ & $D_{0}$ & References \\
\hline $\mathrm{Al}$ & $0(72)$ & 49 & 49 & 49 & 121 & $120.4-144.4$ & $0.035-1.7$ & $40-45$ and 48 \\
\hline $\mathrm{Li}^{*}$ & -6 & 35 & 53 & 47 & 124 & 126 & 0.35 & 61 \\
\hline $\mathrm{Sr}^{*}$ & 71 & $<10$ & 123 & 91 & 92 & & & \\
\hline $\mathrm{Ca}^{*}$ & 21 & 10 & 92 & 52 & 104 & & & \\
\hline $\mathrm{Yb}^{*}$ & 12 & $<10$ & 93 & 47 & 107 & & & \\
\hline $\mathrm{Eu}^{*}$ & 43 & $<10$ & 111 & 64 & 93 & & & \\
\hline $\mathrm{Sc}$ & -12 & 64 & 59 & 41 & 147 & 173 & 5.31 & 62 \\
\hline $\mathrm{Lu}^{*}$ & -1 & 45 & 72 & 34 & 118 & & & \\
\hline $\mathrm{Tm}^{*}$ & 2 & 40 & 75 & 35 & 110 & & & \\
\hline $\mathrm{Er}^{*}$ & 3 & 37 & 75 & 38 & 106 & & & \\
\hline $\mathrm{Ho}^{*}$ & 5 & 34 & 78 & 35 & 102 & & & \\
\hline Dy* & 7 & 31 & 80 & 40 & 106 & & & \\
\hline $\mathrm{Y}^{*}$ & 8 & 30 & 81 & 38 & 101 & & & \\
\hline $\mathrm{Tb}^{*}$ & 10 & 28 & 81 & 38 & 100 & & & \\
\hline $\mathrm{Gd}^{*}$ & 12 & 25 & 83 & 40 & 99 & & & \\
\hline $\mathrm{Sm}^{*}$ & 19 & 17 & 91 & 44 & 97 & & & \\
\hline $\mathrm{Pm}^{*}$ & 22 & 14 & & 45 & 94 & & & \\
\hline $\mathrm{Nd}^{*}$ & 28 & $<10$ & & 48 & 92 & & & \\
\hline $\operatorname{Pr}^{*}$ & 33 & $<10$ & & 52 & 88 & & & \\
\hline $\mathrm{Ce}^{*}$ & 41 & $<10$ & & 56 & 86 & & & \\
\hline $\mathrm{La}^{*}$ & 54 & $<10$ & & 60 & 78 & & & \\
\hline $\mathrm{Zr}$ & -19 & 91 & 52 & 26 & 182 & 242 & 728 & 63 \\
\hline Hf & -21 & 103 & & & 196 & & & \\
\hline $\mathrm{Ti}$ & -25 & 111 & & & 208 & 260 & 1120 & 57 \\
\hline Ta & -29 & 158 & & & 259 & & & \\
\hline $\mathrm{Nb}$ & -28 & 146 & & & 246 & & & \\
\hline V & -24 & 141 & & & 238 & 302.5 & $1.16 \times 10^{4}$ & 57 \\
\hline $\mathrm{V}^{(M)}$ & -24 & 127 & & & 223 & & & \\
\hline W & -23 & 192 & & & 287 & & & \\
\hline Mo & -23 & 172 & & & 267 & 250 & 14 & 64 \\
\hline $\mathrm{Cr}$ & -18 & 152 & & & 241 & $253 ; 261.9$ & 1850 & 65 and 66 \\
\hline $\mathrm{Cr}^{(M)}$ & -13 & 115 & & & 200 & & & \\
\hline $\mathrm{Re}$ & -11 & 193 & & & 276 & & & \\
\hline $\mathrm{Tc}$ & -11 & 175 & & & 258 & & & \\
\hline $\mathrm{Mn}$ & -9 & 147 & & & 228 & $211.4-229$ & $104-1275$ & 65 and $67-69$ \\
\hline $\mathrm{Mn}^{(M)}$ & -6 & 90 & & & 168 & & & \\
\hline $\mathrm{Fe}$ & -2 & 131 & & & 205 & $183.4-258.7$ & $53-9.1 \times 10^{5}$ & 65 and $70-73$ \\
\hline $\mathrm{Ru}$ & -1 & 153 & & & 226 & & & \\
\hline Os & 2 & 169 & & & 239 & & & \\
\hline Co & 1 & 109 & & & 180 & $169-175.7$ & $141-506$ & 66 and $74-76$ \\
\hline $\mathrm{Rh}$ & 5 & 116 & & & 184 & & & \\
\hline Ir & 9 & 175 & & & 192 & & & \\
\hline
\end{tabular}


TABLE V. (Continued.)

\begin{tabular}{|c|c|c|c|c|c|c|c|c|}
\hline \multirow[b]{2}{*}{ Solute } & \multicolumn{5}{|c|}{ Calculated } & \multicolumn{3}{|c|}{ Experimental } \\
\hline & $H_{\text {bind }}^{\square @ X}$ & $H_{m}^{X}$ & $H_{m}^{\mathrm{Al}(1)}$ & $H_{m}^{\mathrm{Al}(3)}$ & $Q$ & $Q$ & $D_{0}$ & References \\
\hline $\mathrm{Ni}$ & 4 & 77 & & & 144 & $144.6 ; 145.8$ & 4.4 & 65 and 74 \\
\hline $\mathrm{Pt}$ & 14 & 86 & & & 144 & & & \\
\hline $\mathrm{Pd}$ & 7 & 74 & & & 139 & & & \\
\hline $\mathrm{Au}^{*}$ & 15 & 37 & 47 & 62 & 103 & $113-121$ & $0.077-0.27$ & $\begin{array}{l}66,70, \text { and } 72 \\
66,70,77 \text {, and }\end{array}$ \\
\hline $\mathrm{Ag}^{*}$ & 9 & 36 & 50 & 57 & 113 & $116.5-118.9$ & $0.12-0.16$ & $\begin{array}{c}78 \\
65,66,77 \text {, and }\end{array}$ \\
\hline $\mathrm{Cu}^{*}$ & -0 & 42 & 35 & 52 & 114 & 133.9-136.1 & $0.06-20$ & 79 \\
\hline $\mathrm{Mg}^{*}$ & 1 & 38 & 61 & 46 & 117 & $120.5 ; 130.4$ & 1.24 & 65 and 80 \\
\hline $\mathrm{Hg}^{*}$ & 25 & 12 & 64 & 64 & 111 & 141.8 & 15.3 & 58 \\
\hline $\mathrm{Cd}^{*}$ & 15 & 24 & 64 & 57 & 114 & 124.3 & 1.04 & 70 \\
\hline $\mathrm{Zn}^{*}$ & 4 & 26 & 50 & 56 & 118 & $116.1-121.4$ & $0.16-0.325$ & $\begin{array}{c}65-67,74, \text { and } \\
81-85\end{array}$ \\
\hline $\mathrm{Tl}^{*}$ & 34 & 9 & 74 & 67 & 105 & 152.7 & 116 & 58 \\
\hline $\mathrm{In}^{*}$ & 21 & 24 & 70 & 58 & 109 & $115.6 ; 122.7$ & $0.123 ; 1.16$ & 86 and 87 \\
\hline $\mathrm{Ga}^{*}$ & 7 & 30 & 52 & 55 & 117 & 122.4 & 0.41 & 66 \\
\hline $\mathrm{Pb}^{*}$ & 39 & 11 & 78 & 67 & 100 & 145.6 & 50 & 58 \\
\hline $\mathrm{Sn}^{*}$ & 25 & 25 & 68 & 59 & 106 & $118.6 ; 119.3$ & $0.245 ; 0.84$ & 86 and 88 \\
\hline $\mathrm{Ge}^{*}$ & 11 & 33 & 55 & 58 & 116 & 121.3 & 0.481 & 66 \\
\hline $\mathrm{Si}^{*}$ & 5 & 44 & 46 & 54 & 113 & $117.6-136$ & $0.35 ; 2.02$ & 65,89 , and 90 \\
\hline $\mathrm{Bi}^{*}$ & 42 & 15 & 79 & 69 & 99 & & & \\
\hline $\mathrm{Sb}^{*}$ & 30 & 24 & 72 & 63 & 105 & 121.7 & 0.09 & 78 \\
\hline
\end{tabular}

Transition metals (TMs), however, behave completely different. No separation between TM from the fourth, fifth, or sixth row in the periodic table can be seen in Fig. 3. In this regard they differ completely from the semimetals. Moreover, TMs appear to generally repel vacancies regardless of their atomic volume. The strongest repulsion occurs for TMs with about the same partial molar volume as Al itself, those

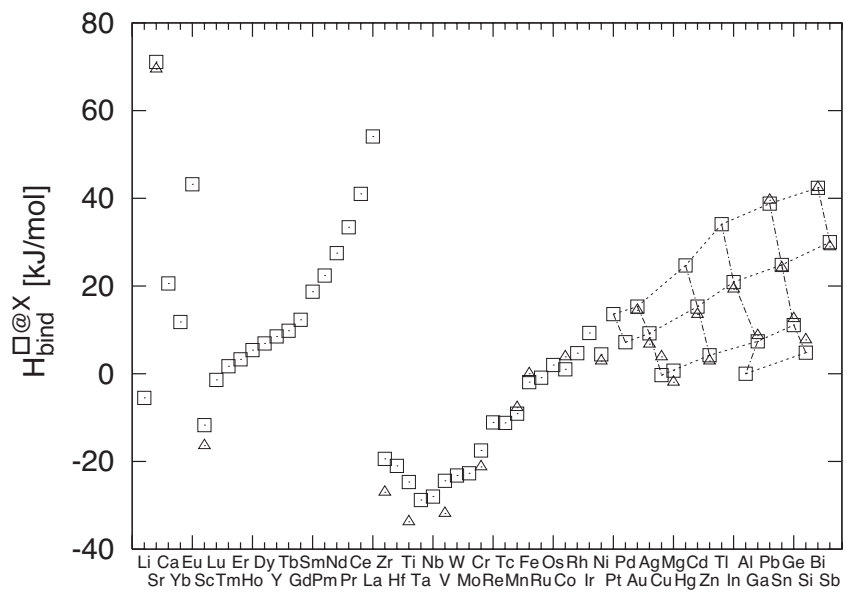

FIG. 2. Solute-vacancy binding enthalpy. Squares: current calculations; triangles: computations of Wolverton (Ref. 19). Lines emphasize tendencies for late transition metals and semimetals. Dashed (dashed-dotted) lines connect squares pertaining to elements in the same columns (rows) of the periodic system. in column $5 \mathrm{a}$ in the periodic table, $\mathrm{V}, \mathrm{Nb}$, and Ta, see Fig. 3. In the $\mathrm{Al}$ matrix, $\mathrm{Co}, \mathrm{Fe}, \mathrm{Mn}$, and $\mathrm{Cr}$ have all smaller volumes than $\mathrm{V}$ (and $\mathrm{Al}$ ) but these elements exhibit less vacancy repulsion than $\mathrm{V}$. The smallest ones among them, $\mathrm{Co}$ and $\mathrm{Fe}$, do not interact significantly with vacancies at all. In other words, while all other solute elements exhibit stronger vacancy binding with increasing partial molar volume, TM solutes in the sequence V-Co exhibit stronger vacancy repulsion as their atomic size increases. It is apparent that TM solutes and vacancies do not interact through a strain caused by volume changes. Earlier, the distinct behavior of $3 d$ TMs in $\mathrm{Al}$ in comparison to other solutes in $\mathrm{Al}$ was pointed out by Hoshino et al. ${ }^{51}$ in calculations where relaxation was ne-

TABLE VI. Computed enthalpy change $H^{\mathrm{nsp}}-H^{\mathrm{sp}}$ in $\mathrm{kJ} / \mathrm{mol}$ and magnetization $m$ in $\mu_{B}$ as a result of spin polarization in fully relaxed $\mathrm{Al}_{63} X$, in $\mathrm{Al}_{62} \square X$ in the initial state (solute $X$ next to a vacancy $\square$ ) and $\mathrm{Al}_{62} \square X \uparrow$ in the transition state for solute-vacancy interchange for $X=\mathrm{V}, \mathrm{Cr}$, and $\mathrm{Mn}$; nsp: non-spin-polarized; sp: spin-polarized calculation.

\begin{tabular}{|c|c|c|c|c|c|c|}
\hline & \multicolumn{2}{|l|}{$X=\mathrm{V}$} & \multicolumn{2}{|c|}{$X=\mathrm{Cr}$} & \multicolumn{2}{|c|}{$X=\mathrm{Mn}$} \\
\hline & $H^{\mathrm{nsp}}-H^{\mathrm{sp}}$ & $m$ & $H^{\mathrm{nsp}}-H^{\mathrm{sp}}$ & $m$ & $H^{\mathrm{nsp}}-H^{\mathrm{sp}}$ & $m$ \\
\hline $\mathrm{Al}_{63} X$ & 0.0 & 0.0 & 11.3 & 2.2 & 16.3 & 2.1 \\
\hline $\mathrm{Al}_{62} \square X$ & 0.5 & 1.0 & 15.9 & 2.5 & 19.7 & 2.5 \\
\hline $\mathrm{Al}_{62} \square X \uparrow$ & 15.0 & 2.2 & 52.8 & 2.9 & 76.3 & 3.3 \\
\hline
\end{tabular}




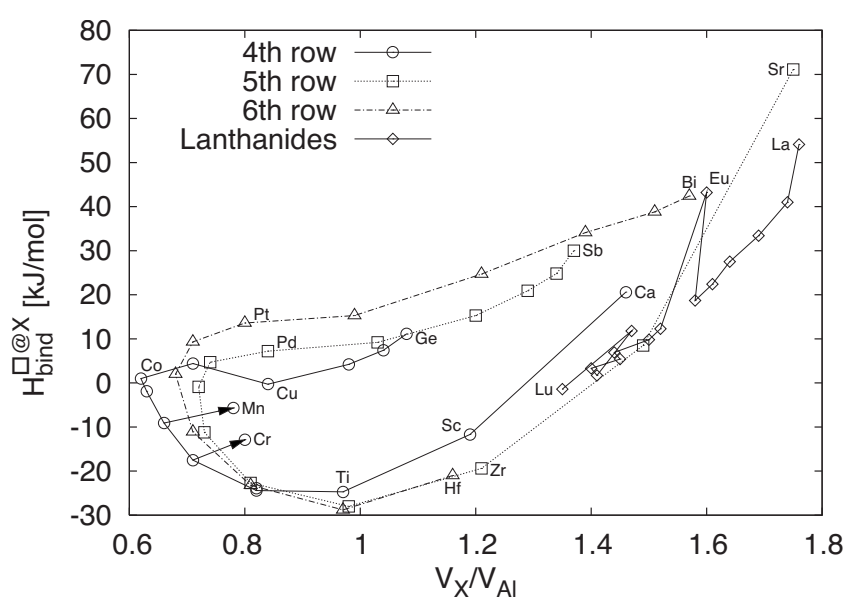

FIG. 3. Solute-vacancy binding enthalpy as a function of relative partial molar volume of the solute. The arrows point from the non-spin-polarized toward the spin-polarized results for $\mathrm{Cr}$ and $\mathrm{Mn}$.

glected, and more recently by Sandberg and Holmestad ${ }^{6}$ and Mantina. ${ }^{7}$ Hoshino et al..$^{51}$ attributed the strong TM- $\square$ repulsion to the strong Al-TM bond. After all, when a vacancy far from a TM solute atom is moved next to it, the energy change is due to trading an Al-TM bond for an Al-Al bond. The strong Al:sp-TM: $d$ hybridization is strongest for about half-filled $d$ bands and is negligible for nearly empty or filled $d$ bands. This explains why the strongest Al-TM bonds occur around column $6 \mathrm{a}(\mathrm{Cr}, \mathrm{Mo}$, and $\mathrm{W})$. It is then also apparent that the TM- $\square$ interchange transition state should be particularly unfavorable because then there are the least number of favorable Al-TM bonds. The highest activation barriers occur for TM with about half-filled $d$ bands because these have the strongest Al-TM bonds. It also explains that spin polarization for $\mathrm{Cr}$ and $\mathrm{Mn}$ should reduce both TM- $\square$ interchange barriers and the TM- $\square$ repulsion; spin polarization causes the half-filled non-spin-polarized $d$-like bands to split in an almost filled majority-spin $d$-like band and an almost empty minority-spin $d$-like band. This same effect was also recognized in other phenomena determined by Al-TM bonding, such as site preference of magnetic TM impurities in aluminides. ${ }^{52}$ Sandberg and Holmestad ${ }^{6}$ pointed to a strong correlation between the residual resistivity and the diffusion activation energy for TM impurities in Al. This is completely in keeping with the impurity scattering description, where $d$-like impurity states centered around the Fermi energy (i.e., of TMs with half-filled $d$ bands) are the most effective in reducing the density of states at the Fermi energy while simultaneously being efficient scatterers. ${ }^{53}$ This line of reasoning also suggests that elements that do not hybridize and form localized bonds might have lower migration barriers. Normal "free-electron" metals, such as $\mathrm{Li}, \mathrm{Mg}, \mathrm{Zn}, \mathrm{Cu}$, and $\mathrm{Ag}$, and even $\mathrm{Si}$ in a high coordinated metallic environment, that have similar atomic sizes as $\mathrm{Al}$, all have weak vacancy binding only and migration barriers that are fairly close to that of $\mathrm{Al}$ itself, as expected from the nondirectional metallic bonding.

The rare-earth (RE) series show very pronounced trends; the first half of the series strongly attracts vacancies and the more strongly the earlier in the series; the migration barriers

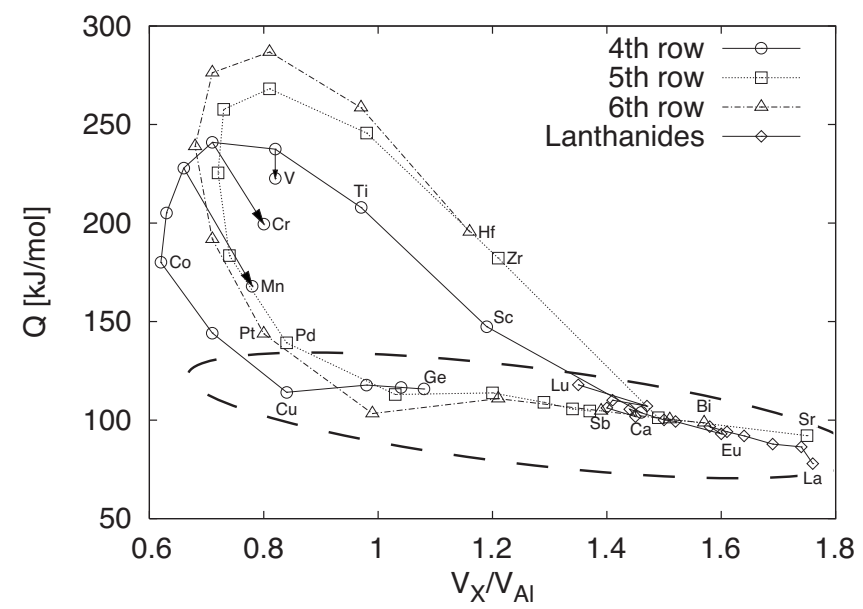

FIG. 4. Diffusion activation energy $Q$, computed with Eq. (7), as a function of relative partial molar volume of the solute. The arrows point from the non-spin-polarized toward the spin-polarized results for $\mathrm{Cr}, \mathrm{Mn}$, and $\mathrm{V}$. The area within the dashed oval highlights the elements that obey an approximately linear correlation between partial molar volume in $\mathrm{Al}$ and $Q$.

are lower than that for $\mathrm{Al}$ self-diffusion. These trends can be understood when it is taken into account that the Al sp-like and $\mathrm{RE} f$-like electronic states do not hybridize significantly. Therefore, there are no strong Al-RE bonds, unlike in the example of TM. In the absence of such localized bonds, relaxation plays a dominant role just as we have encountered earlier for Sr. The large atomic size early in the RE series causes strong vacancy binding and the formation of solutevacancy complex with a small barrier for solute-vacancy interchange. The late RE solutes are smaller and therefore have only weak vacancy binding.

Like the solute- $\square$ binding enthalpy, the diffusion activation energy $Q$ correlates well with partial molar volume as shown in Fig. 4; the series $\mathrm{Cu}-\mathrm{Ge}, \mathrm{Pd}-\mathrm{Sb}$, and $\mathrm{Pt}-\mathrm{Bi}$ have $Q$ values close to that of Al self-diffusion, with the larger solutes, at the end of the series, having somewhat lower $Q$ values than the smaller solutes at the beginning of the series. The lanthanides, with large partial molar volumes especially at the beginning of the series, have activation energies that are lower than that for Al self-diffusion. Again, the TMs follow a completely different trend with a pronounced maximum for TMs in the middle of the series, at the 6a column: $\mathrm{Cr}, \mathrm{Mo}$, and W. A much weaker tendency is that TMs from the sixth row of the periodic table $(5 \mathrm{~d})$ have about the same or slightly higher $Q$ than the corresponding TM from the fifth row (4d) and that TMs from the fourth row (3d) have lower $Q$ 's than elements in the same column from the fifth and sixth rows. The $Q$ values for TMs can differ very strongly from the value found for Al self-diffusion; TMs in columns 5a and 6a have diffusion activation energies that are twice or more than that for Al self-diffusion. This is apparent in Fig. 5 also where the pronounced maximum for $Q$ stands out among computed as well as experimentally determined values. It should be noted that for large non-TM solutes, such as $\mathrm{Sr}, Q$ is determined by the rate of the Al- $\square$ interchange, as evident from Eq. (7). This explains the rather weak variation in $Q$ among non-TMs as the variation is due mostly to the 

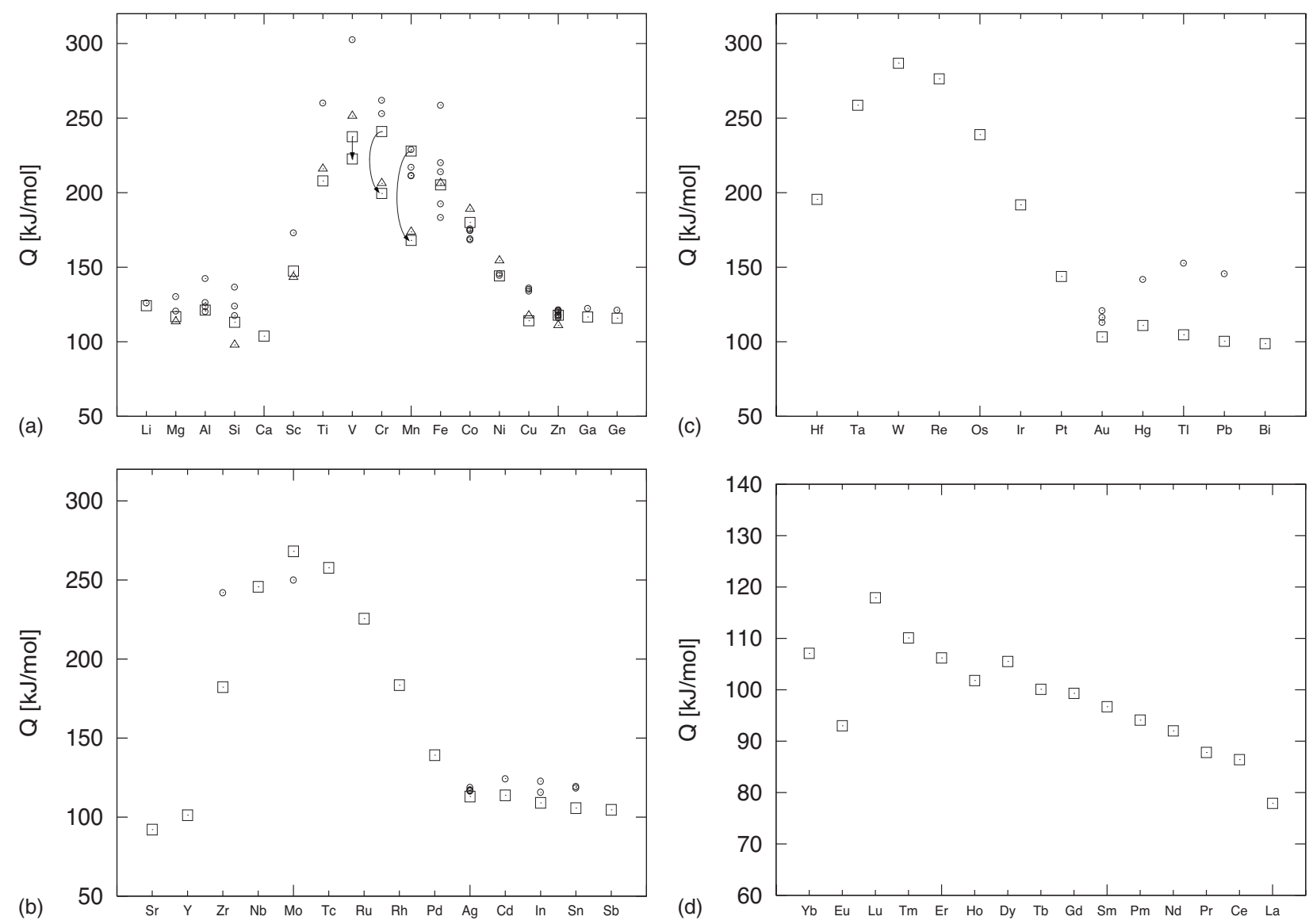

FIG. 5. Diffusion activation energy $Q$ for impurities in Al. Lanthanides are ordered according to their Mendeleev number (Refs. 49 and 50). Squares: current calculations; triangles: computations of Sandberg and Holmestad (Ref. 6); circles: experimental data (specifically mentioned in Table V). For $\mathrm{V}, \mathrm{Cr}$, and $\mathrm{Mn}$ an arrow points from the non-spin-polarized to the spin-polarized results.

variation in the vacancy binding energy. The shaded area in Fig. 4 shows the strong linear correlation between partial molar volume and $Q$. It follows roughly that

$$
Q_{X} \approx Q_{\mathrm{Al}}\left[1+0.3\left(1-\frac{V_{X}}{V_{\mathrm{Al}}}\right)\right],
$$

where $V_{X}$ is the partial molar volume of solute $X, V_{\mathrm{Al}}$ is the volume per $\mathrm{Al}$ atom, and $Q_{\mathrm{Al}}$ is the activation energy for $\mathrm{Al}$ self-diffusion.

Figure 5 allows for an easy comparison with previous theoretical calculations and with experimental data. Although there is a wide spread in the experimental data, our computed results generally agree fairly well with the lower experimental values. The agreement with the previous Sandberg and Holmestad $^{6}$ results is also quite good although our numbers are systematically lower. It is very likely that our lower values are connected with our decision to perform calculations at the experimental lattice parameter pertaining to about 600 $\mathrm{K}$. We also find $\mathrm{Cr}$ and $\mathrm{Mn}$ to be magnetic, and we find a similar reduction in $Q$. We find $\mathrm{V}$ to be magnetic in the vicinity of a vacancy only, and the effect on the diffusion activation energy is much smaller than that for $\mathrm{Cr}$ and $\mathrm{Mn}$. Contrary to Sandberg and Holmestad, ${ }^{6}$ but in agreement with Mantina, ${ }^{7}$ we did not find $\mathrm{Fe}$ to exhibit spin polarization in Al. Other impurities that we closely examined for possible magnetic behavior, such as $\mathrm{Co}, \mathrm{Nd}, \mathrm{Sm}, \mathrm{Eu}$, and $\mathrm{Yb}$, could not be stabilized in the spin-polarized state. This might be connected to our expanded lattice parameter, although lattice expansion usually stabilizes the magnetic state, ${ }^{53}$ and there is a strong connection with local positional relaxation as well. Experimentally there is no evidence for spin-polarization behavior of $\mathrm{Fe}$ or $\mathrm{V}$ in $\mathrm{Al}$, and only $\mathrm{Cr}$ and $\mathrm{Mn}$ are found to be spin polarized. ${ }^{54}$ While in previous unrelaxed calculations $\mathrm{Fe}$ has been found to be magnetic, ${ }^{51}$ other fully relaxed calculations have also reported no spin polarization. ${ }^{55,56}$ Regardless of what the magnetic state may be at $T=0 \mathrm{~K}$, we find that non-spin-polarized results for $\mathrm{V}, \mathrm{Cr}$, and $\mathrm{Mn}$ agree much better with experiment than the spin-polarized results. Possibly, magnetic effects are quenched at the temperatures that are relevant experimentally, or the magnetic state associated with the transition state does not fully develop during the actual transition process. This might imply a mild violation of the Born-Oppenheimer approximation at the transition state. While the nonmagnetic calculations for $\mathrm{Cr}$ and $\mathrm{Mn}$ give a fair agreement with the experimental $Q$, the same is not the case for V. In view of the large spread in the experimental $Q$ values for the early TM impurities and the exceptionally large experimental value of $302.5 \mathrm{~kJ} / \mathrm{mol}$, possibly the experimental value ${ }^{57}$ is not accurate.

Our calculated $Q$ values for other early TM impurities deviate significantly from the experimental values also. Al- 
though we find large $Q$ 's, typically the experimental values are still much larger $(\mathrm{Sc}, \mathrm{Ti}$, and $\mathrm{Zr}$ ). Possibly, for these slowly diffusing impurities other mechanisms play an important role, such as through divacancies or via other lattice imperfections. This will remain a topic for future study. For the remaining TMs the computed $Q$ 's are typically within the spread of the experimental data (Mo, Fe, Co, and Ni). The noble metals have well-established experimental $Q$ 's within a rather narrow range. The values computed by us are typically 5-15 kJ/mol lower $(\mathrm{Cu}, \mathrm{Ag}$, and $\mathrm{Au})$, which appears to be a fair agreement.

The $Q$ computed for non-TMs agree generally quite well with the experimental numbers, but here our calculations also tend to give values at the lower end of the spectrum ( $\mathrm{Li}$, $\mathrm{Mg}, \mathrm{Zn}, \mathrm{Cd}, \mathrm{Ga}, \mathrm{In}, \mathrm{Si}, \mathrm{Ge}, \mathrm{Sn}$, and $\mathrm{Sb}$ ). For the heavy elements $\mathrm{Hg}, \mathrm{Tl}$, and $\mathrm{Pb}$ our computed values are significantly lower than the experimental $Q$ 's. ${ }^{58}$ We are suspicious of these experimental values ${ }^{58}$ because the experimental diffusion prefactors $D_{0}$ are anomalous as well. The diffusion rate of non-TM solutes is dominated by the rate of Alvacancy interchanges, and therefore we must expect that the diffusion prefactor is generally close to that for $\mathrm{Al}$ selfdiffusion, of the order of $0.1 \times 10^{-4} \mathrm{~m}^{2} \mathrm{~s}^{-1}$. Indeed, the available experimental measurements for non-TM solutes range from $0.1 \times 10^{-4}$ to $2 \times 10^{-4} \mathrm{~m}^{2} \mathrm{~s}^{-1}$, with those of $\mathrm{Hg}$, $\mathrm{Tl}$, and $\mathrm{Pb}^{58}$ as sole exceptions with $D_{0}=15.3 \times 10^{-4}, 116$ $\times 10^{-4}$, and $50 \times 10^{-4} \mathrm{~m}^{2} \mathrm{~s}^{-1}$, respectively. These large experimental $D_{0}$ values compensate the large experimental $Q$ values. Our computed $Q$ values suggest that $\mathrm{Hg}, \mathrm{Tl}$, and $\mathrm{Pb}$ follow the trends seen in non-TMs for partial molar volume with vacancy binding and activation energy, and suggest a $D_{0}$ value in line with other non-TMs.

\section{CONCLUSION}

We have presented calculations of the solute-vacancy binding enthalpy and the diffusion activation energy for a large number of solutes in fcc aluminum. Results were compared with experimental data and with other theoretical calculations for about half of the solutes considered here. Generally there is a fair agreement although our values tend to be at the low side of the experimental spectrum. The somewhat low diffusion activation energies are believed to be a consequence of the neglect of anharmonic effects in the calculations. $^{3,6}$ It has been shown that the vacancy binding and diffusion activation energies of TMs to the left of $\mathrm{Cu}$, $\mathrm{Ag}$, and $\mathrm{Au}$ in the periodic table differ strongly from those of all other elements. The non-TM solutes in aluminum feature vacancy binding that is strongly correlated with their partial molar volume in relation to that of $\mathrm{Al}$, with the larger solute atoms exhibiting stronger vacancy binding than smaller solute atoms. For non-TM solutes the diffusion activation energy is correlated even better with partial molar volume, with the larger solute atoms having lower activation energy than the smaller solute atoms. However, the diffusion activation energy of non-TM solutes is always rather close to and often lower than that for Al self-diffusion, typically within the window of $75-125 \mathrm{~kJ} / \mathrm{mol}$, with the lowest values occurring for the early lanthanides. The diffusion rate of non-TM solutes was dominated by the rate of Al-vacancy interchanges, and therefore we predict that the diffusion prefactor is generally also close to that for Al self-diffusion, of the order of 1 $\times 10^{-5} \mathrm{~m}^{2} \mathrm{~s}^{-1}$. Most of the available experimental measurements for non-TM solutes bears this out, as they range from $0.1 \times 10^{-4}$ to $2 \times 10^{-4} \mathrm{~m}^{2} \mathrm{~s}^{-1}$. The experimental diffusion activation energies and diffusion prefactors for $\mathrm{Hg}, \mathrm{Tl}$, and $\mathrm{Pb}$ break trends seen for similar elements and therefore are suspected to be in error.

TMs to the left of $\mathrm{Ni}, \mathrm{Pd}$, and $\mathrm{Pt}$ in the periodic table do not follow the partial molar volume trends described above. TMs, especially those early in the series, strongly repel vacancies, regardless of their partial molar volume relative to that of $\mathrm{Al}$. The strongest vacancy repulsion occurs for the $5 \mathrm{a}$ column in the periodic table, $\mathrm{V}, \mathrm{Nb}$, and $\mathrm{Ta}$, but repulsion is also strong for the $4 \mathrm{a}$ and $6 \mathrm{a}$ columns, with typical values in the range of $20-30 \mathrm{~kJ} / \mathrm{mol}$. The diffusion activation energies for TMs are larger than that for Al self-diffusion. The largest values appear for TMs in column 6a, Cr, Mo, and W, and exceed twice the activation energy for Al self-diffusion. Our calculations reveal that TM solute-vacancy interchanges are the rate determining process for diffusion, and therefore we expect diffusion prefactors to deviate much from that for $\mathrm{Al}$ self-diffusion. The experimental data bear this out with reported prefactors being 1-6 orders of magnitude greater. Strong bonding due to $s p$ - $d$ electronic hybridization between $\mathrm{Al}$ and TM impurities explains these features. It should be remarked that in concentrated Al-TM alloys the bonds are not strongest in the middle of the TM series, ${ }^{59}$ which suggests that the diffusion activation energy for TM impurities may vary strongly with the impurity concentration.

Spin polarization is found for $\mathrm{Cr}$ and $\mathrm{Mn}$ where, particularly in the transition state, it causes a large reduction in the energy. Therefore, the diffusion activation energy is strongly reduced by spin polarization. $\mathrm{V}$ exhibits spin polarization only near a vacancy, and the energetic effect is small. By comparison the effect of spin polarization on the vacancy binding is rather modest. The experimental data agree much better with the non-spin-polarized results for $\mathrm{V}, \mathrm{Cr}$, and $\mathrm{Mn}$. This suggests that at temperature ranges of interest for diffusion the spin polarization does not play a role.

The lanthanides show clear tendencies, especially when the elements are ordered according to the Mendeleev number of Pettifor; ${ }^{49,50}$ early lanthanides such as La and Ce strongly attract vacancies because of their large size and the nondirectional nature of the Al-lanthanide bond. Therefore, the early lanthanides have very low migration energies for solute-vacancy interchanges, just as $\mathrm{Ca}$ and $\mathrm{Sr}$, and the $\mathrm{Al}-$ vacancy interchange is rate determining for solute diffusion. As a result, the diffusion activation energy of lanthanides follows the same trend as the vacancy binding, with the early lanthanides exhibiting rather low activation energies of about $80 \mathrm{~kJ} / \mathrm{mol}$ and the later lanthanides approaching the activation energy for $\mathrm{Al}$ self-diffusion. $\mathrm{Eu}$ is a trend breaker in the lanthanide series, in keeping with its well-known chemical characteristics known as the europium anomaly.

The general tendencies found in this study for bulk solute diffusion properties for a large number of metallic solutes in Al suggest that for other modes of solute transport through Al such as pipe diffusion through dislocation cores and 
grain-boundary diffusion, very broad generalizations are also possible. Moreover, solute diffusion through other normal metals with nearly-free-electron character derived from $s p$-like states such as $\mathrm{Ga}, \mathrm{Sn}$, and $\mathrm{Sb}$ might follow a similar classification in TM and non-TM behavior.

\section{ACKNOWLEDGMENTS}

The authors acknowledge support from the Netherlands
Institute for Metals Research ${ }^{91}$ in collaboration with the Foundation for Fundamental Research on Matter (FOM) of the Netherlands under Project No. 02EMM032 and from the Materials Innovation Institute ${ }^{92}$ under Project No. MC4.05212. The National Computer Facilities of the Netherlands Foundation for Scientific Research (NWO) are gratefully acknowledged for computer resources provided under Grant No. SH-007-07.
${ }^{1}$ L. Mondolfo, Aluminum Alloys: Structure and Properties (Butterworths, London, 1976).

${ }^{2}$ A. LeClaire and G. Neumann, in Diffusion in Solid Metals and Alloys, Landolt-Börnstein: Group III Condensed Matter, edited by H. Mehrer (Springer, New York, 1990), Vol. 26, pp. 151-155.

${ }^{3}$ N. Sandberg, B. Magyari-Köpe, and T. R. Mattsson, Phys. Rev. Lett. 89, 065901 (2002).

${ }^{4}$ M. Mantina, Y. Wang, R. Arroyave, L. Q. Chen, Z. K. Liu, and C. Wolverton, Phys. Rev. Lett. 100, 215901 (2008).

${ }^{5}$ A. Van der Ven and G. Ceder, Phys. Rev. Lett. 94, 045901 (2005).

${ }^{6}$ N. Sandberg and R. Holmestad, Phys. Rev. B 73, 014108 (2006).

${ }^{7}$ M. Mantina, Ph.D. thesis, College of Earth and Mineral Sciences, Materials Science and Engineering, The Pennsylvania State University, 2008.

${ }^{8}$ G. Henkelman, B. P. Uberuaga, and H. Jónsson, J. Chem. Phys. 113, 9901 (2000).

${ }^{9}$ G. Henkelman and H. Jónsson, J. Chem. Phys. 113, 9978 (2000).

${ }^{10}$ P. Hohenberg and W. Kohn, Phys. Rev. 136, B864 (1964).

${ }^{11}$ W. Kohn and L. J. Sham, Phys. Rev. 140, A1133 (1965).

${ }^{12}$ G. H. Vineyard and G. J. Dienes, Phys. Rev. 93, 265 (1954).

${ }^{13}$ G. Vineyard, J. Phys. Chem. Solids 3, 121 (1957).

${ }^{14}$ D. D. Fontaine, Solid State Physics (Academic, New York, 1994), Vol. 47, pp. 33-176.

${ }^{15}$ A. Zunger, in Statics and Dynamics of Alloy Phase Transitions, NATO Advanced Studies Institute Series B: Physics, edited by P. Turchi and A. Gonis (Plenum, New York, 1994), pp. 361-419.

${ }^{16}$ M. Sluiter, D. de Fontaine, X. Q. Guo, R. Podloucky, and A. J. Freeman, Phys. Rev. B 42, 10460 (1990).

${ }^{17}$ S. Curtarolo, D. Morgan, and G. Ceder, CALPHAD: Comput. Coupling Phase Diagrams Thermochem. 29, 163 (2005).

${ }^{18}$ W. Frank, C. Elsässer, and M. Fähnle, Phys. Rev. Lett. 74, 1791 (1995).

${ }^{19}$ C. Wolverton, Acta Mater. 55, 5867 (2007).

${ }^{20}$ T. G. Stoebe and H. I. Dawson, Phys. Rev. 166, 621 (1968).

${ }^{21}$ K. W. Kehr, K. Binder, and S. M. Reulein, Phys. Rev. B 39, 4891 (1989)

${ }^{22}$ A. Lidiard, Philos. Mag. 46, 1218 (1955).

${ }^{23}$ A. LeClaire and A. Lidiard, Philos. Mag. 1, 518 (1956).

${ }^{24}$ A. D. Le Claire, J. Nucl. Mater. 69-70, 70 (1978).

${ }^{25}$ M. H. F. Sluiter and Y. Kawazoe, Modell. Simul. Mater. Sci. Eng. 8, 221 (2000).

${ }^{26}$ Y. Mishin, M. R. Sorensen, and A. F. Voter, Philos. Mag. A 81, 2591 (2001).
${ }^{27}$ M. S. Daw, Comput. Mater. Sci. 38, 293 (2006).

${ }^{28}$ J. P. Perdew, K. Burke, and M. Ernzerhof, Phys. Rev. Lett. 77, 3865 (1996).

${ }^{29}$ G. Kresse and J. Furthmüller, Phys. Rev. B 54, 11169 (1996).

${ }^{30}$ G. Kresse and J. Furthmüller, Comput. Mater. Sci. 6, 15 (1996).

${ }^{31}$ P. E. Blöchl, O. Jepsen, and O. K. Andersen, Phys. Rev. B 49, 16223 (1994).

${ }^{32}$ G. Kresse and D. Joubert, Phys. Rev. B 59, 1758 (1999).

${ }^{33} \mathrm{G}$. Kresse, http://cms.mpi.univie.ac.at/vasp-workshop/slides/ pseudoppdatabase.pdf

${ }^{34}$ M. C. Gao, A. D. Rollett, and M. Widom, Phys. Rev. B 75, 174120 (2007).

${ }^{35}$ H. J. Monkhorst and J. D. Pack, Phys. Rev. B 13, 5188 (1976).

${ }^{36}$ W. Frank, C. Elsässer, and M. Fähnle, Phys. Rev. Lett. 74, 1791 (1995).

${ }^{37}$ K. Parlinski, Z. Q. Li, and Y. Kawazoe, Phys. Rev. Lett. 78, 4063 (1997).

${ }^{38}$ M. H. F. Sluiter, M. Weinert, and Y. Kawazoe, Europhys. Lett. 43, 183 (1998).

${ }^{39}$ P. Ehrhart, in Atomic Defects in Metals, Landolt-Börnstein: Group III Condensed Matter, edited by O. Madelung (Springer, New York, 1991), Vol. 25, p. 213.

${ }^{40}$ T. S. Lundy and J. F. Murdock, J. Appl. Phys. 33, 1671 (1962).

${ }^{41}$ M. Beyeler and Y. Adda, J. Phys. (Paris) 29, 345 (1968).

${ }^{42}$ T. Volin and R. Balluffi, Phys. Status Solidi 25, 163 (1968).

${ }^{43}$ E. Fradin, Ph.D. thesis, University of Illinois, 1971.

${ }^{44}$ R. Messer, S. Dais, and D. Wolf, Proceedings of the 18th Ampere Congress, edited by P. Allen, E. Andrew, and C. Bates (Nottingham Ampere Committee, Nottingham, England, 1974).

${ }^{45} \mathrm{G}$. Hood, in Solute-Defect Interaction: Theory and Experiment, edited by S. Saimoto, G. Purdy, and G. Kidson (Pergamon, New York, 1985), p. 83.

${ }^{46}$ S. Kurth, J. P. Perdew, and P. Blaha, Int. J. Quantum Chem. 75, 889 (1999).

${ }^{47}$ K. Carling, G. Wahnström, T. R. Mattsson, A. E. Mattsson, N. Sandberg, and G. Grimvall, Phys. Rev. Lett. 85, 3862 (2000).

${ }^{48}$ F. Y. Fradin and T. J. Rowland, Appl. Phys. Lett. 11, 207 (1967).

${ }^{49}$ D. G. Pettifor, Solid State Commun. 51, 31 (1984).

${ }^{50}$ D. G. Pettifor, J. Phys.: Condens. Matter 15, V13 (2003).

${ }^{51}$ T. Hoshino, R. Zeller, and P. H. Dederichs, Phys. Rev. B 53 8971 (1996).

${ }^{52}$ M. Sluiter, M. Takahashi, and Y. Kawazoe, Acta Mater. 44, 209 (1996).

${ }^{53}$ G. Trambly de Laissardière, D. Nguyen-Manh, and D. Mayou, Prog. Mater. Sci. 50, 679 (2005).

${ }^{54}$ D. Riegel and K. D. Gross, Physica B 163, 678 (1990). 
${ }^{55}$ D. Guenzburger and D. E. Ellis, Phys. Rev. Lett. 67, 3832 (1991).

${ }^{56}$ P. G. Gonzales, L. A. Terrazos, H. M. Petrilli, and S. FrotaPessôa, Phys. Rev. B 57, 7004 (1998).

${ }^{57}$ D. Bergner, Neue Hütte 29, 207 (1984).

${ }^{58}$ F. Sawanayagi and R. Hasiguti, J. Jpn. Inst. Met. 42, 1155 (1978).

${ }^{59}$ F. de Boer, R. Boom, W. Mattens, A. Miedema, and A. Niessen, in Cohesion in Metals: Transition Metal Alloys, edited by F. de Boer and D. Pettifor (North-Holland, Amsterdam, 1988).

${ }^{60}$ K. Wang and R. Reeber, Philos. Mag. A 80, 1629 (2000).

${ }^{61}$ Y. Minamino, T. Yamane, and H. Araki, Metall. Trans. A 18, 1536 (1987)

${ }^{62}$ S.-I. Fujikawa, in DIMAT-96, Defect and Diffusion Forum, edited by H. Mehrer, C. Herzig, N. Stolwijk, and H. Bracht (ttp, Switzerland, 1997), Vol. 143-147, pp. 115-120.

${ }^{63}$ T. Marumo, S. Fujikawa, and K.-I. Hirano, J. Jpn. Inst. Met. 23, 17 (1973).

${ }^{64}$ L. Barr and E. Smith, in DIMETA-82, Diffusion in Metals and Alloys, edited by F. Kedves and D. Beke (ttp, Switzerland, 1983), Vol. 66-69, p. 325.

${ }^{65}$ Y. Du, Y. A. Chang, B. Huang, W. Gong, Z. Jin, H. Xu, Z. Yuan, Y. Liu, Y. He, and F. Y. Xie, Mater. Sci. Eng., A 363, 140 (2003).

${ }^{66}$ N. L. Peterson and S. J. Rothman, Phys. Rev. B 1, 3264 (1970).

${ }^{67}$ D. Bergner and E. Cyrener, Neue Hütte 18, 9 (1973).

${ }^{68}$ G. M. Hood and R. J. Schultz, Philos. Mag. 23, 1479 (1971).

${ }^{69}$ S. Fujikawa and K. Hirano, in Age-Hardenable Aluminium Alloys, Materials Science Forum, edited by I. Kovacs and J. Lendvai (ttp, Switzerland, 1987), Vol. 13-14, pp. 539-546.

${ }^{70}$ W. B. Alexander and L. M. Slifkin, Phys. Rev. B 1, 3274 (1970).

${ }^{71}$ G. M. Hood, Philos. Mag. 21, 305 (1970).

${ }^{72}$ D. L. Beke, I. Gödény, I. Szabo, G. Erdélyi, and F. J. Kedves, Philos. Mag. A 55, 425 (1987).

${ }^{73}$ C. Becker, G. Erdélyi, G. Hood, and H. Mehrer, in DIMETA-88, edited by F. Kedves and D. Beke (ttp, Switzerland, 1990), Vol. 66-69, pp. 409-414.

${ }^{74}$ G. Erdélyi, D. L. Beke, F. J. Kedves, and I. Godény, Philos. Mag. B 38, 445 (1978).

${ }^{75}$ M. S. Anand and R. P. Agarwala, Philos. Mag. 26, 297 (1972).

${ }^{76}$ G. M. Hood, R. J. Schultz, and J. Armstrong, Philos. Mag. A 47, 775 (1983).

${ }^{77}$ M. Beyeler, F. Maurice, and R. Seguin, Mém. Sci. Rev. Métall. 67, 295 (1970).

${ }^{78}$ S. Badrinarayanan and H. Mathur, Int. J. Appl. Radiat. Isot. 19, 353 (1968).

${ }^{79}$ S. Fujikawa and K. Hirano, in DIMETA-88, Defect and Diffusion Forum, edited by F. Kedves and D. Beke (ttp, Switzerland, 1990), Vol. 66-69, pp. 457-452.

${ }^{80}$ S. Rothman, N. Peterson, L. Nowicki, and L. Robinson, Phys. Status Solidi B 63, K29 (1974).

${ }^{81}$ I. Gödény, D. Beke, and F. Kedves, Phys. Status Solidi A 13, K155 (1972).

${ }^{82}$ S. Fujikawa and K.-I. Hirano, Trans. Jpn. Inst. Met. 17, 809 (1976).

${ }^{83}$ D. Beke, I. Gödény, F. Kedves, and G. Groma, Acta Metall. 25, 539 (1977).

${ }^{84}$ N. L. Peterson and S. J. Rothman, Phys. Rev. B 17, 4666 (1978).

${ }^{85}$ D. L. Beke, I. Gödény, and F. J. Kedves, Philos. Mag. A 47, 281 (1983).

${ }^{86}$ M. S. Anand and R. P. Agarwala, Phys. Status Solidi A 1, K41 (1970).

${ }^{87}$ G. M. Hood and R. J. Schultz, Phys. Rev. B 4, 2339 (1971).

${ }^{88}$ G. Erdélyi, C. Freitag, and H. Mehrer, Philos. Mag. A 63, 1167 (1991).

${ }^{89}$ D. Bergner and E. Cyrener, Neue Hütte 18, 356 (1973).

${ }^{90}$ S.-I. Fujikawa, K.-I. Hirano, and Y. Fukushima, Metall. Trans. A 9, 1811 (1978).

91 www.nimr.nl

${ }^{92}$ www.m2i.nl 